\title{
Oil Spill Detection Using Multi Remote Piloted Aircraft for the Environmental Monitoring of Sea Aquatorium
}

\author{
Aleksandrs URBAHS ${ }^{1 \dagger}$, Vladislavs ZAVTKEVICS ${ }^{2 *}$ \\ ${ }^{1,2}$ Institute of Aeronautics, Riga Technical University, Viskalu iela 36A, Riga, LV-1006, Latvia
}

\begin{abstract}
The objective is to carry out an analysis of remote oil spill detection using multi Remote Piloted Aircraft (RPA). A multi-RPA system provides reliable detection of an oil spill with significant advantages over other existing single RPA methods. The objective is to develop an algorithm of using multi-RPA for the monitoring of oil spills. In this paper centralized and multi-RPA algorithms for oil spill detection are analysed.
\end{abstract}

Keywords - Environmental monitoring; multi Remote Piloted Aircraft system; oil spill detection; sea aquatorium.

\section{INTRODUCTION}

Pollution of the world's sea aquatorium with oil products is one of the most urgent environmental problems today. An average of 13-14 million tonnes of oil products enters the world's oceans annually.

The ecological state of the Baltic Sea is also a source of serious concern for environmental specialists. The main reasons are an increase in oil pollution as a result of the expansion of the offshore oil and gas industry and an increase in the traffic of ships. With the increase in the volume of transportation of oil products, the risk of pollution of the marine area by oil products increases.

Synthetic Aperture Radar (SAR) is the most effective and basic satellite sensor because of its wide area of monitoring and 24-hour surveillance [1]. The main direction for the development and the improvement of the methods of remote monitoring of oil pollution using satellites is the abandonment of photographic methods and transition to the use of new multi-spectral and hyperspectral sensors in a payload. Satellite-based SARs today are an important oil spill monitoring tool for covering large areas, both day and night, in all weather conditions [2]. Despite the considerable advantage compared to other surveillance systems, satellite systems have limitations of the frequency of filming the same sea area (the frequency is every 3 hours, considering the possibility of changing the viewing angles). High definition radiolocation data is provided to the responsible organizations with a delay, related to delivery to the receiver. The production of a map, based on radiolocation reception data, can take $1-$ 2 days from the moment the oil pollution has occurred. During pollution elimination, these conditions lead to the need for operational monitoring by mobile vehicles.

Aerial surveillance is also widely used for oil pollution monitoring. Aerial monitoring of oil pollution is an effective method of controlling the state of the sea aquatorium. The main

* Corresponding author.

E-mail address: vladz@inbox.lv 
problem with the use of piloted aircraft is their limited use due to weather conditions (cloudiness and fog). In addition, the use of pilot-operated aircraft is characterized by a relatively long response time (up to 3 hours) and very high operating costs [1]. Aerial filming using piloted aircrafts does not always allow the detection of signs of an oil spill. The accuracy of the assessment depends on the sea surface fluctuation, intensity of light and observation angles. It is not possible to simultaneously and completely cover the area of monitoring of the sea aquatorium. In most cased, the thickness of the oil film cannot be determined by satellite measurements. During the monitoring of oil pollution using airplanes and helicopters, the thickness of the oil film can be roughly determined by using microwave and infrared radiometers. Automatic systems are also used to control the marine pollution of the marine environment. Automatic marine systems represent a complex, which is fixed by an anchor and has a payload. In connection with the large number of shortcomings of remote sensing and stationary automatic systems, accurate results and analysis can be obtained only with the use of an integrated system. The marine observational system is a complex of observations conducted from various observation platforms by unified measuring instruments on the basis of a single methodology and a legal base [3]. This system consists of two subsystems: ground and remote.

The ground subsystem includes the sea shore hydrometeorological observation network, a vessel observation network set up by the monitoring program, oceanographic observation and operation network, automatic buoy stations, anchored and drifting network, the national marine pollution monitoring administration network. Buoys and underwater vehicles are installed and operated taking into account maritime safety requirements, and, therefore, information on their location is required [3]. The space subsystem consists of a group of meteorological and specialized satellites that allow monitoring of the water surface. The main disadvantage of this is the stationary location of buoys, drifting in areas with high traffic of ships and fishing, which reduces maritime safety. Unfortunately, in case of an oil spill, the system provides information with a delay.

After detection of oil spills using remote sensing, it is necessary to monitor the spill with ships, which allows determining both the dynamics of the distribution of pollutants in the seawater and the type of oil product. In addition, the analysis and visual inspection of remote sensing data depends on a variety of factors, including the specifics of the detectors, synoptic situation, solar illumination and even the human factor of the observer [30]. Response ships may be used as a part of the surveillance system and a platform for the RPAs which will improve oil slick detection capability in the vicinity of ship operation area [1]. Mobile marine platforms, in contrast to remote sensing tools, provide an opportunity to take samples from the water surface. The main disadvantage of using vessels for monitoring is their low speed: the average speed is 15 miles per hour and the maximum speed is 30 miles per hour.

The RPAs are used to perform different environmental monitoring tasks due to high actuality of obtained data. The RPAs offer remotely sensed data at a high spatial resolution, a flexible local control for vehicles that is fuel efficient and an operation with minimum visual intrusion [1]. The implementation of the RPAs platform for detecting oil pollution can minimize surveillance cost and improve data actuality. Small RPA potentially offer a low-cost alternative to conventional remote sensing platforms, and research to date shows promise for this technique [4]. Usually, surveillance activities include monitoring of large sea areas. Single RPAs cannot perform all tasks in a large area due to limited flight endurance. In order to solve this problem, a system with interaction of multiple RPAs is needed. 
Monitoring of oil pollution requires continuous monitoring for 24 hours, based on a well-designed distribution of remote sensing vehicles. The monitoring system should be remote, distributed, multisensory and automated.

The use of the RPA for monitoring of marine oil pollution will help to eliminate the disadvantages of existing remote sensing equipment, including increasing reliability of automated monitoring systems for oil pollution detection. Some monitoring organizations offer to use a remote airplane to provide more cost-effective solutions to response personnel [5]. But there are currently no cheap commercial systems capable of providing reliable remote sensing with high sensitivity to oil pollution. Using a payload of the RPA with different sensor types will significantly increase the reliability and accuracy of measurable contaminant parameters. The automatic system using the RPA will ensure that the following tasks are resolved:

- Surveillance of large marine areas;

- Continuous monitoring of marine ecosystems;

- Monitoring of the spreading of oil spill dynamically;

- Sampling using specially designed RPA for pollution identification [6];

- Determination of the type of contamination product;

- Finding and collecting evidence of oil pollution from ships;

- Provision of operational information to the responsible services in the event of an oil pollution accident.

The main problem of oil pollution monitoring is the need to monitor the sea surface, covering large areas with variable geometry, and to control the large number of ships with different parameters (at variable speed, trajectory) [7]. This could mean that the management approach may involve developing new environmental performance evaluation tools for the industry to understand more about the negative impacts of their production processes [29]. The main task here is to develop the RPA route optimization algorithm, in which the RPA has the ability to continuously adjust the flight parameters according to the real situation and dynamically changing control parameters. There are two approaches to solving the problem of route optimization of target points using the minimum time of optimization criteria. This problem should be solved in real time due to sea monitoring specificity. In this case, all objects of interest will be present in a matrix with dynamic and static objects. Within this matrix, coordinates of dynamic objects, such as an oil spill, will be calculated in real time [7].

The first approach is to find an optimal route using the Dijkstra algorithm on a graph. The main approach for finding an optimal decision between nodes is solving the problem with Dijkstra's algorithm. Dijkstra's algorithm includes several heuristics to direct the vehicle towards the goal state and help to eliminate unnecessary computations by removing any unnecessary state analysis [8]. The algorithm is related to heuristic methods. In heuristic methods, it is recommended to use some natural selection rules to select elements for the solution. The time of this algorithm is equal to:

$$
\Theta(E)=\Theta\left(V^{2}\right)
$$

where

E Edges;

V Vertices.

Most fixed target path planning problems can be considered similar to the traveling salesman problem (TSP) that has been evaluated significantly in numerous ways for decades since Dantzig et al. [9], developed a solution as an integer linear program. 
Linear programming methods can be implemented only for a small number of static objects [7]. This algorithm has high accuracy but needs a lot of time to find the shortest route. Routing of robots and unmanned vehicles, the heuristic algorithms can be used.

The second approach is using the multi-agent system with decentralized control of agents. Usually, the architecture of the system is the same as in biogenic systems, such as swarm and others. Its strengths lie in the autonomy of the activities of separate RPA, possibility of educating the units and collective communication system. The ant colony algorithm is one of the most successful methods using the swarm intelligence [10].

\section{Methods ANd Procedures}

The main objectives of this research include an analysis of the possibilities of applying a group of RPA for oil spill monitoring, mathematical modelling of monitoring procedure for various object matrices, development of an algorithm for searching an optimal flight route for the monitoring of objects, as well as creation of special monitoring tools.

The main objective of the multi-RPA system is detection and following of an oil spill, taking into account movement and parameters, and their changing during time. The mission of multi-RPA includes three scenarios: the coordinates of an oil spill are known, the RPA perform the monitoring of sea aquatorium for the presence of an oil spill, the RPA perform monitoring of spreading of an oil spill and changing parameters such as oil film thickness. The main requirement is that the task should be solved in real time. The task can be presented as a multi-agent monitoring mission of different objects. Multi-agents are RPA with payloads for oil pollution detection. All RPA have visible and near-infrared (VNIR), thermal and multispectral cameras in their payloads. Each agent has a characteristic capability limited by sensors and, due to this, should perform monitoring of a defined targets subset. The application of the multi-RPA system allows making effective planning of resources in the sea spatial and time frames. Considering dynamical changes of an oil spill, this approach will help in case of immediate changes in a situation to make new planning of a flight trajectory.

The multi-agent system has more advantages than a single RPA. The main advantage is splitting of tasks between a team of the RPA. During the monitoring of a large area of sea aquatorium, a single RPA cannot perform a mission due to restrictions. These restrictions are:

- In the monitoring area there are two or more oil spills;

- Due to the long distance of a route, flight missions will be performed using an autopilot. When information about an oil spill will be suddenly received, the RPA will be sent to monitoring only from a zone covered by a ground station;

- Duration of the oil spill surveillance mission is limited by RPA endurance;

- Application of a single RPA allows using only one type of platform for monitoring. In this case, an oil spill will be monitored by one platform, which makes it impossible to effectively use different payloads. For example, fixed wing platforms with long autonomy cannot be used for oil spill sampling [6];

- Information about the detected pollution can be transmitted in a zone covered by a ground station.

Specifically, UAV swarms "must be controllable by non-specialist operators whose primary job is something other than controlling the UAV" [11]. Taking into account a large amount of static and dynamic objects in the sea aquatorium, the multi-RPA should be controlled by an operation system. For a system such as swarm with multi-RPA, the action of mobile units performing the surveillance should be organized using standardized rules. The oil spill detection mission includes surveillance of large areas for oil spills performed by multi-RPA. 
The approach of multi-agent control in this case should be based on a multilevel control system. The multilevel system consists of two levels, a high level and a low level. The high level receives global information and information about static and dynamic objects from the AIS (Automated Identification System). The architecture of this system allows controlling swarm activities within the territory of a monitoring area without communicating with agents. The main advantage is the ability to assign a task for the RPA before they have left the area of communication and are performing objective of the mission without information about the condition of individual agents. On the high level, different algorithms of organizing the activities of the multi-RPA can be compared and the optimal evaluated solution for surveillance can be implemented. In addition, this architecture can use optimization algorithms of visiting objects of interest with the calculation of the position of a dynamic object. The target of the dynamic environment can be represented as points with such main parameters as initial speed and course [7]. On the high level, after analysing all input information on a multilayer chart, the position of the center and shape of the swarm will be determined.

The high-level controller should (loosely speaking) optimize the dynamics of the swarm (i.e., position and velocity), its shape, dimension(s), and motion with respect to objectives of the mission within the scenario characterized by the absence-presence of a mission operator [12].

The high-level management of the RPA allows performing control in areas with normal communication and in areas where communication with the RPA can be lost. This type of architecture provides the ability to change a decision at each stage to that, which is optimal. In this system the RPA can effectively perform both types of tasks: high level tasks and low-level monitoring tasks. Hierarchical logic for decision making must be implemented to ensure the most effective completion of the mission and rapid response to mission or system changes [13].

There is an approach for the swarm control using knowledge about input parameters. This type of control system uses three types of knowledge: a global layer, a local layer and the condition of RPA agents [14]. The use of this approach constructs a global layer using all possible input data from an external system.

One of the conceptions of system architecture includes four-level control [15]. Separate controls are defined at each level: decision making, planning a route of agents, planning of each object of interest and possibility to exchange an agent which is disabled due to a technical failure. The advantage of this system architecture is the possibility to implement the redundancy principle when there is a risk of losing the RPA.

One of the most effective methods of controlling a group of RPA is a method based on the application of a multi-level control system [13]-[15]. However, the proposed approach is applicable in case of controlling the RPA when moving towards the dedicated area without taking account the flight path of object monitoring. Taking into account that the main objective is to minimize the cost of the system and time of solving for control decisions, the control system should include three levels: decision making, planning a route of agents, planning of each object of interest. The control of the multi-RPA is to be organized by using flexibility for moving mobile ground stations. Considering the dynamic environment, there should be a possibility to implement immediate response based on information at the global level. It is very important in case of emergency pollution to implement centralized control and assign a task based on only a little knowledge. During the time while this type of task is being performed, knowledge received from agents will be analysed and new generated task will be assigned for followers. For example, after receiving information about a pollution 
event from a responsible organization, two or more RPA will be sent to the dedicated area. After performing initial surveillance, an operator of the centralized system on the higher level will assign a task for the RPA equipped with sampling devices or other payloads [16]. Higher levels of autonomy require proper automation management strategies in order to effectively lessen the operator load while avoiding automation-related problems, such as automation bias, complacency and reduced situation awareness [17].

Implementation of the high-level control allows changing the area and position of the swarm after receiving visual information from an agent. Implementation of the gravitation method for finding the center mass will be used for the optimization of RPA position. This application allows organizing communication between the low level and the high level with feedback. All modules of the low level should be connected to the high level with the possibility of interconnection between them. The surveillance data received by the swarm RPA processing gives an opportunity to make a decision at the higher level.

The oil spill detection mission can be presented on the high level with areas with high, medium and low risk of oil pollution. To improve the effectiveness of the system, the principle of center of gravity can be applied. A large area of the sea aquatorium can be split into areas with different risks of pollution. An area can typically include static objects and dynamic objects (ships). A mass center can be calculated for each area. After determining subareas on the high level, the gravity centers of subareas and the gravity center of the area will be calculated.

This structure of the system allows simplifying low-level tasks. These tasks include only navigation to the objects prescribed by the high-level system specialized algorithm for the trajectory of an object and sensors for monitoring. The multi-RPA exhibit autonomy when the system software is capable of making - and is entrusted to make - substantial real-time decisions, without human involvement or supervision [18].

Splitting to subtasks with a limited number of variables is a way of reducing the swarm shape to a "small" manageable set from the computation standpoint, as well as from the point of maintaining full capability of management [19]. On the high level, a spatial environment that is defined by a closed circuit should be developed by the controlling system. For oil spill detection, this environment will be created using dedicated algorithms. Considering the specificity of the mission on the high level, after receiving real-time information about pollution, the RPA in the swarm can be split into subgroups to perform tasks effectively. In this case, information from a sensor of one RPA will be transmitted to the group members. The model of oil pollution spreading allows sending information to the RPA to navigate to the position. These positions will be calculated based on the probability and corrected after receiving information from the sensors of the RPA. This strategy allows improving efficiency of a group of the RPA. Using the information received from RPA sensors on the high level, the model of oil spreading will be recalculated. The position obtained from the recalculated model of oil spreading on the surface of water will be sent to the dedicated RPA group. The flight cost for each RPA will be calculated on the high level, based on the received knowledge. The cost of RPA flight can be estimated based on the length of flight trajectory required for the surveillance of the defined area. On the high level, after the recalculation of oil spreading model, using special algorithms for surveillance, the trajectory length for the surveillance of an object will be calculated. This high-level management strategy gives an opportunity to monitor the border of the oil film on the water surface in real time.

After analysing oil pollution incidents in the sea aquatorium, a control cycle for multi-RPA will be developed. This control cycle will be implemented on the high level. This is a multistage cycle. The first stage is responsibility to the oil pollution incident. On the high 
level, systems are receiving real time information from the agents of RPA, satellite remote sensing systems, information about ships in the area. An oil slick is an emergency event. The architecture of the system allows providing discrete event control. The second stage is dynamical planning. On this stage on the high level, formulation of tasks and replanning are performed. The third stage is the agreement and revision of tasks and control states in real time. At this stage, there is a possibility of shifting tasks in time. It means that when external information about an oil slick is received, multi-RPA assigned for the low-level risk of pollution area can be redirected to areas near the oil spill. The fourth stage includes monitoring and checking the performance of assigned tasks. The task of planning an oil pollution monitoring mission is very difficult because it depends on many external factors. Some external factors such as wave height, oil temperature and water salinity cannot be identified. For the successful performance of planning on the high level, a possibility to implement centralized control is needed. The centralized control allows performing correction activities. In non-standard situations manual corrections of activity planning on the high level can be implemented. For oil pollution monitoring mission, it is necessary to implement a decision support system. The decision support system allows checking different control plans during planning, using information received in real time.

The task of distribution of targets and planning of a mission for each RPA has a variety of solutions. The main parameters for solving are: distribution of objects between the RPA, a flight plan of object monitoring for a single RPA, time of target monitoring. Due to this, the main optimization criterion is the time of performing object monitoring.

For a dynamically changing system, such as a large area of sea aquatorium with many ships, dynamical programming principle can be used - splitting a task to a subset for minimizing the solving time. The planning of an oil spill detection mission can be split into three stages. The first stage is distribution of objects between the multi-RPA. The objective of this stage is to create a set of RPA routes with minimal time between the starting and end points on the graph, taking into account that there are multi RPAs. The second stage is creating an optimal route for the surveillance of the targets for each RPA. This task can be represented by a graph where verticals are objects, edges are time cost. This task can be solved using the dynamic programming method and the branch and bound method. The third stage is determining the target type, application of monitoring flight trajectory for typical targets and possible additional trajectory if needed.

The first stage can be represented as operation of multi-RPA during the monitoring of the matrix of targets. A system includes $n$ RPA, $n>1$, and a control system. The matrix of targets can be represented as a set of objects which are represented by using geographic coordinates. The objective can be formulated as finding $\mathrm{m}$ of points from the set of points $N$ that provide minimal flight time for each RPA [7]:

$$
T=\min _{m<N} i \sum_{i=1}^{m} T_{i},
$$

where $T_{i}$ is flight time for each RPA.

At this stage, a matrix of objects with time cost should be created.

After creating the matrix of objects, the multi-RPA will be split to single RPA.

On the high level, the planning of routes for multi-RPA should be performed for each group of objects. A target of the dynamical environment can be represented as points with such main parameters as initial speed and course. Targets are divided into four groups with speed $V=0,0<V<14,14<V<23$, and $23<V$ (in knots) [7]. Objects with speed $V=0$ are static 
objects. For static objects, the main task is to visit an object for surveillance or take a sample [6]. Objects with speed $V>0$ are dynamic objects. The algorithm for these types of targets includes monitoring a target with known motion parameters. To represent the moving object, the traverse sailing method is used. This method allows computing the position of the object as well as the course and distance to the next point. In this case, the distance to a sea target from the starting point is calculated using the following formula:

where

$$
D=V \cdot t
$$

$V \quad$ Speed of a ship in knots;

$t \quad$ Time.

For surface objects with dynamic characteristics, speed and vector of motion can be calculated using external factors, such as wind, height of waves and current. During a surveillance mission for detecting illegal and emergency oil spills, the task can be formulated as a search of a target with unknown geographic coordinates or probability location. There is an approach for dividing the area into grid cells and assigning them for each multi-agent. The main disadvantage of this approach is long time of search in case of using centralized control mode. The best results with reduced time of search will be achieved when using probability evaluation. A robust RPA search method for determining target existence is proposed with consideration that the prior probabilities for a given cell are poorly known [20]. The number of search patterns will be defined using the distribution function. This approach has a disadvantage which is low precision. For environmental missions, high quality data is needed to perform a developed program. Overall pollution as an environmental hazard, cannot be addressed only locally, local actions are irreplaceable and vitally necessary, but only through global actions we can expect the so much needed results and impact for better quality of life, and healthier population [32]. There is a method for tracking the boundary of an oil spill using model predictive control and universal kriging. Universal kriging is an interpolation technique closely related to regression analysis [21].

There is a method of decentralized cooperative control of multiple-RPA performing multiple tasks in an urban environment [22]. In this algorithm, each agent uses its own field of view and analyses this information comparing it with data received from agents that are near. After processing information, a flight plan for an agent to search in the next grid will be generated. This algorithm needs an agent that is equipped with information retranslation equipment; this condition reduces reliability of communication. The decentralized algorithm needs more time for determining a search plan of a flight due to the presence of big amount of external information.

This method needs significant computation system resources and due to this fact cannot be used on the low level of control system. For the multi-agent system to engage in oil spill detection, an algorithm was developed.

Oil spill detection actions by multi-RPA can be presented by variables $C_{t}$ - coordinates on plane of the RPA, $O_{t i}$ position of $i$ point of the border of an oil spill. Taking into account that determining the border of clean water and oil spill consists of discrete events on the high level of the system, a control decision $U_{t i}$ should be created for RPA $i$. The procedure of finding the optimal control (the decision) is based on the analysis of the recurrence relation:

$$
f_{n-1}\left(S_{l}\right)=\operatorname{optimum}\left[R_{l+1}\left(S_{l}, U_{l+1}\right)+f_{n-(l+1)}\left(S_{l+1}\right)\right], l=\overline{0, n-1},
$$


where

$\begin{array}{ll}U_{1}=u_{l}^{1}, \ldots, u_{l}^{m} & \text { Solution chosen at the } 1^{\text {st }} \text { step; } \\ S_{1}=s_{l}^{1}, \ldots, s_{l}^{m} & \text { State of the system at the } 1^{\text {st }} \text { step; } \\ R_{1} & \text { Immediate effect achieved at the } 1^{\text {st }} \text { step; } \\ f_{n-1} & \text { Optimal value of the effect achieved for the } n-1 \text { steps; } \\ n & \begin{array}{l}\text { Number of steps (stages) [7]; } \\ S_{t i}\end{array} \\ & \begin{array}{l}\text { Discrete event, the moment when the point of the border of the oil spill is } \\ \text { found by the PRA. }\end{array}\end{array}$

The control decision found on the high level and obtained on the high level is actually the next waypoint of the RPA trajectory on the low level. In defined time intervals, a command which takes into account the probability of finding a new point of oil slick border is generated on the high level of the system. This is required because during the spread of an oil spill on the water surface, oil slicks are usually divided into smaller parts.

The decision is found taking into account the matrix of the observed points of the slick border and of the current detected point $S_{t i}$. The decision $U_{t i}$ for a single RPA on the high level depends on external information. When information is received from the satellite monitoring system or from a calculated mathematical model, a vector of the RPA will last from present coordinates of the RPA to the probable location. After detecting the first point of the border of an oil spill, the RPA starts collecting information about the current detected point $\mathrm{S}_{t i}$. The main principle of controlling the RPA model in vector form is:

$$
\dot{x}=f(x, u, t)
$$

where

$\mathrm{t} \in\left[t_{0}, t_{\mathrm{k}}\right]$

$x\left(t_{0}\right)=x_{0}[7]$

$x=\left(x_{1}, x_{2}, \ldots, x_{\mathrm{n}}\right)$

$f_{u}=\left(u_{1}, u_{2}, \ldots, u_{m}\right)$

Vector of RPA condition (the coordinate vector);

$f=\left(f_{1}, f_{2}, \ldots, f_{n}\right)$

Controlling vector;

$\left[t_{0}, t_{k}\right]$

Vector function of their arguments;

Time interval during which the RPA flight is performed [7].

At this stage, the control command depends on the detected coordinates of a border point.

In an emergency situation, the coordinates of oil spill border should be defined. This is very important information for organizations responsible for eliminating oil spills and maximally effective use of resources for cleaning. The border will be defined using such criteria as clean water and oil slick. The detection of an oil slick will be performed on the low level by the RPA controller. For this purpose, the algorithm for each type of sensor of payload will be used. It is needed due to the properties of an oil slick; the best results will be received using payload with integrated sensors. During the processing of data from the sensor, the algorithm provides a signal 'yes' in case of clean water or 'no' in case of dirty water. After receiving a signal 'yes', the RPA starts expanding its square search. During this search, the distance between legs is defined by the field of view of the sensors. The trajectory of expanding square search will be completed when the detection signal 'yes' is received on the length of a leg more than $100 \mathrm{~m}$. After that, the RPA will be redirected to another position outside the checked square, using the algorithm. The direction of motion to a new position will be on a line between the registered changing signal from 'yes' to 'no'. In this case, a group of RPA is performing a task of finding a border of static objects. With the centralized approach, an agent dispatcher 
will assign a task, such as surveillance of areas which depend on the number of RPA. At the initial stage, the center of gravity will be in the detection point. During the time of receiving information from the RPA, the center of gravity will be recalculated. In this algorithm, when the RPA finds some areas of the border of an oil slick, these coordinates can be used to calculate the probability of spreading.

The area of monitoring can be presented as a surface area which includes all types of targets. To perform the task, there is a set of RPA equipped with sensors and located in points with geographic coordinates, where $\mathrm{R}$ is a number of all RPA that can be used Fig. 1.

$$
R=\sum_{i=1} R_{i}
$$

The objective of detecting oil pollution should be solved using restrictions because it has a lot of solutions.

Taking into account that usually this algorithm is implemented for one agent, targets should be distributed between the RPAs. This will be done by an operator which will split surface areas into subareas. In a subarea, targets are represented as a closed graph.

On the high level, the system is solving the problem of object distribution between the RPAs. This task is assigning targets for a set of RPAs. This problem can be solved using centralized control on the high level. The area of sea aquatorium has many types of objects for oil pollution monitoring. For the minimization of system capacity and solution time, the area will be split into subareas. The center of gravity of objects will be calculated for each subarea. This approach allows representing the task of visiting objects as a graph on the high level.

This problem can be represented as a graph with two parts. One part of the graph is represented by a set of RPA $R=\{0,1, \ldots, m\}$. The second part of the graph is the set of subareas $S=\{0,1, \ldots, n\}$. Each subarea has weight $w_{\mathrm{k}} k=0, n$. The weight of a subarea is presented by the time cost of visiting all types of objects inside. The time cost will be calculated as the time spent visiting all objects from the gravity center of a subarea and the time cost of monitoring all objects. To determine the weight of an edge, the following formula is used:

$$
w_{i j}=t_{\text {flightij }}+t_{\text {surveillance }}
$$

where

$t_{\text {flightij }}$

$t_{\text {surveillance }}$

Flight time from the previous object;

Duration of the object surveillance mission [7].

The set of edges connected to the RPA and subareas will be used for finding a solution on the high level.

This problem can be represented as a graph with two parts. One part of the graph is represented by a set of RPA $R=\{0,1, \ldots, m\}$. The second part of the graph is the set of subareas $S=\{0,1, \ldots, n\}$. Each subarea has a weight $w_{k} k=0, n$. The weight of a subarea is presented by the time cost of visiting all types of objects inside. The time cost will be calculated as the time spent visiting all objects from the gravity center of a subarea and the time cost of monitoring all objects. To determine the weight of an edge, the Eq. (7) is used. The set of edges connected to the RPA and subareas will be used for finding a solution on the high level. 


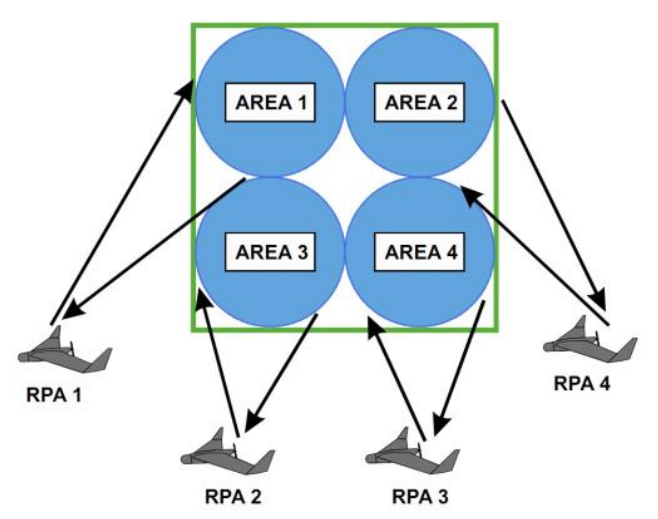

Fig. 1. A multi-RPA system for oil spill detection.

To improve effectiveness and minimize the time of solving the routing of multi-RPA on the high level, dedicated algorithms were developed. The types of monitoring are divided into five categories: initial search of oil pollution, monitoring of point objects, monitoring of line objects, monitoring of surface objects, surveillance of dynamic surface objects. For these types of oil pollution surveillance, a data base with a trajectory and time of performing the task was created. A route plan of the RPA will depend on the tasks set, search method of an object and the mobility, size, visibility and placement density on the field [1].

In the input of algorithm, the following information is received: height of a flight, field of view of sensors, speed of the flight, turning radius, wind direction and coordinates of an object. At the next stage, the width of the surveillance zone and flight trajectory according to object type will be defined. For detailed viewing of separate sea areas within the area of operation straight lines, parallel routes are used [1]. After the orientation of RPA against wind and selection of an appropriate trajectory, a calculation of the turning points of surveillance route for the object will be performed. At this final stage, the length of the route is calculated, as well as the time cost of the task, which is calculated with account of average RPA speed. For the surveillance of static and dynamic objects on the surface, an algorithm with criteria of monitoring quality was developed.

The sea aquatorium is represented as an area with objects (targets). Objects, for the use of a typical monitoring algorithm, are divided into four basic groups:

- Point-based objects;

- Linear objects;

- Surface objects;

- Surface objects with dynamic characteristics (oil spills).

Point-oriented objects are described by geographic or Cartesian coordinates of the center of an object. These are the objects the size which do not go across the viewpoint of the camera at the observation flight altitude.

Linear objects are elongated objects that, in flight, travel along the same path, enter the view of the camera and are characterized by the coordinates of the broken lines of the turning points.

Surface objects are objects constrained by a polygon. Their filming is carried out on several occasions (courses). They are characterized by a polygon surrounding the observation object (the area where water quality monitoring is conducted), coordinates of the vertices. 
Surface objects with dynamic characteristics (oil spills) are objects described by a motion equation. In the input of the algorithm, the following information was sent: range of flight height depending on the RPA platform type, type of payload, payload surveillance parameters, radius of turn, endurance of the RPA. The algorithm received information about the kind of object on the surface - whether it is static or dynamic. At the next stage, surveillance parameters are defined: height of a flight, field of view of sensors, turning radius. At the next stage, the surface is approximately calculated. After that, the surface is divided into small areas. This approach gives the opportunity to satisfy objects with a non-standard perimeter, such as oil spills. After analyzing the form of an object, the optimal trajectory will be chosen from the database. After that, the quality function of a flight during surveillance will be defined. The software allows to check a flight cycle with the help of uninterrupted function intervals after checking the local minima of the function. The length of flight trajectory for an object will be determined by quality criteria. To perform the assigned task, when the payload sensors are switched to surveillance, the speed of flight in calculations is taken as constant with an account of external factors. The shortest trajectory, considering the flight speed, corresponds to the shortest time of the mission. Overlap square is used as the criteria of the quality of the flight for static and dynamic objects. These criteria can be determined after the flight, when the overlap is compared with the square of an object.

The developed method allows solving the problem by using the external information which is received without using the RPA payload. Routine surveillance tasks in cooperation with satellite monitoring system consist of surveillance of sea waters near shore terminals, anchoring area of ports, traffic routes surveillance based on analysing AIS information (especially tankers), and inspection of illegal discharging routes [1].

One RPA will be connected to each subarea. To find the optimal solution, criteria for the minimization of the time of a task performed by multi-RPA will be used. The sum of weights of flights performed by multi-RPA $W=\sum_{0}^{m} w_{i j}$ should be minimized using the target function. This solution will be present when monitoring an area with multi-RPA. The architecture of the system allows recalculating with new data matrix parameters in case of detecting an oil spill and changing the control decision. This approach allows to organize system decomposition by splitting into levels. This architecture gives an opportunity to minimize a set of possible solutions for the oil pollution monitoring task.

In the graph theory, a solution can be found using a solution tree. The graph $\mathrm{G}$ is the tree. The verticals of the graph are nodes of the decision tree. The decision tree includes internal nodes and external nodes (leaf nodes). An internal node is a decision node with two or more branches where a decision will be made regarding the next node of the tree to be visited. Such decision-making uses a decision function. External nodes have no branches and describe the classification of input data or the decision. The solving process starts in the root node because it has no entering edges. In this node, the decision function is calculated. The decision function for the RPA monitoring mission can be represented as time of a flight in a route; this value is fixed at the next node. For example, if in a surface area with the number of targets $N$ the decision tree has $N$ ! external nodes. The main objective is splitting the tree to parallel subsets when looking for a solution. While searching for the solution, the classification of condition will be performed. This algorithm analyses at which node to start, which node to proceed to, and when to find a conclusion. The advantage of this approach is doing classification without many calculations. The decision tree can be represented as a closed graph. 
This algorithm can be implemented only for an RPA as a part of multi-RPA because it is determinated. Long computation time is caused by the necessity of visiting each node for finding a short route path. The results of the performed calculations show that the tree algorithm has a very small accuracy for the number of vertexes greater than five [7]. This algorithm can be used in a multi-agent system with the dedicated condition that there are defined sets of surveillance objects and distributed subsets for each RPA. This can be implemented with an approach using a central monitoring area distribution system.

The main advantage of the algorithm of ant colony is activity criteria pheromone. In the biogenic ant colony system, trails are marked with pheromone when returning to the colony. The main property of pheromone is evaporation with the lapse of time. It means that the ant algorithm can be used for a matrix with objects where there is time for visiting all the objects. In a biogenic ant colony, ants move randomly. It means that pheromone is a probability value and needs movements of the RPA during a long-time window for accuracy.

Positive feedback - high pheromone quantity - provides the optimal shortest route. The negative feedback in this algorithm motivates the RPA to find new solutions with the shortest length. The warm robotics is based on the metaphor of social insects and emphasizes aspects like decentralized control, limited communication between agents, local information, emergence of a global behaviour, and robustness [23]. There is a restriction that before implementing the results for the RPA, a mathematical model has to be created and tested through a number of experiments. The ant algorithm for the RPA can be presented as a set of RPA $R_{m}=\left\{R_{1}, \ldots, R_{m}\right\}$, where $R$ is the RPA $m$ - number of RPA. The objective of the RPA is finding the optimal route. The objective lies in mathematical modelling of behaviour of ants traveling on different routes in different directions with a target to find the optimal route. For this objective, presentation of target matrix as a graph $\mathrm{G}(V, E)$ is used. To find a solution, it is necessary to determine the value of pheromone trail. The route is represented as edges of the graph which connect the vertices of the graph. Taking into account the fact that an RPA traveling on the edge of the graph $\mathrm{E}$ has left some amount of the pheromone, probability of having an optimal route is proportional to the pheromone amount on the edge. This pheromone is connected with the optimality of choice. The pheromone left on the edge $i j$ is evaporated with the lapse of time. Pheromone changes during time $t$ can be presented by the formula:

$$
\tau_{i j} \leftarrow(1-\rho) \tau_{i j}(t)+\Delta \tau_{i j}(t),
$$

where $\rho \in(0,1]$ pheromone trail evaporation coefficient defined by the intensity of activities of the RPA.

$$
\tau_{i j}=\sum_{R=1}^{m} \Delta \tau_{i j}^{R}(t)
$$

where

$R \quad$ Each RPA;

$m \quad$ Number of RPA at each iteration.

The probability of RPA transportation from vertex $i$ to vertex $j$ can be presented by the formula:

$$
P_{i j}(t)=\frac{\tau_{i j}(t)^{\lambda}\left(\frac{1}{t_{i j}}\right)^{\beta}}{\sum \tau_{i j}(t)^{\lambda}\left(\frac{1}{t_{i j}}\right)^{\beta}},
$$


where

$\tau_{i j} \quad$ Pheromone level;

$\lambda, \beta \quad$ Constants which defined the type of solution search;

$\lambda \quad$ Choice of object with minimal time cost;

$\beta \quad$ Solution with pheromone level;

$t_{i j} \quad$ Time cost between vertices $i$ and $j$.

The time cost between vertices is proportional to pheromone quantity on the edge; this means there will be the minimal time cost on the edge with the maximum pheromone quantity. Unfortunately, using only positive feedback based on the maximum content of pheromone will result in the organization of transportation by RPA only on the local optimal route. This disadvantage can be eliminated by using the feedback of pheromone evaporation. The time of pheromone evaporation can be determined experimentally, and it improves the quality of the optimal solution. The main advantage is finding the best solution with a large number of experiments with optimality criteria $\tau$. This property makes it preferable for big dynamic target matrices. The main problem is that for finding a solution, high capacity of system resources is needed. From another point of view, this approach gives an opportunity to find a rational solution.

The proposed method integrates the ant colony algorithm and an analytic hierarchy process that showed good results for path planning using the ant colony algorithm [24].

For time cost matrices with a small number of objects, the tree, Dijkstra and other heuristic algorithms can be used. This algorithm provides more strong points to the multi-RPA system than genetic and neural networks. An oil spill detection mission can be completed by the multi-RPA system using the ant algorithm with a dynamic matrix, in which the position of objects is calculated by using a mathematical model.

\section{RESULTS AND DISCUSSION}

\subsection{Analysis of Algorithms and their Application for Oil Spill Detection}

The evaluation of the algorithm of decision tree and the ant algorithm shows that these can be used for the multi-RPA system. Taking into account the dynamics of the oil spill described by the mathematical model with continuously changing coordinates, an effective monitoring mission can be effectively performed by the RPA $n>1$. The decision tree algorithm is a determined algorithm, but it can be used only for one RPA. In order to use it, firstly, a centralized system where the area is split into subareas for a single RPA should be build. To implement this algorithm, a high level system with two levels of decomposition was designed. The ant algorithm can be used in the decentralized system with a time cost matrix which includes a large number of objects. To analyse the algorithms, an analysis was conducted (Table 1). 


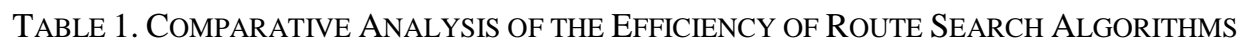

\begin{tabular}{|c|c|c|c|}
\hline Algorithm & Dijkstra & Decision tree & Ant colony \\
\hline Mathematical approach & Heuristic & Determinated & Probabilistic \\
\hline Accuracy & Low & High & Average \\
\hline Number of graph $N$ nodes & $N<40$ & $N<20$ & $N<100$ \\
\hline Number of agents & 1 & 1 & Multi-agent \\
\hline Calculation time & Average & Average for $N>20$ & High for $N>50$ \\
\hline Advantages & For $N<40$ & $\begin{array}{l}\text { For } N<20 \text { : opportunity of } \\
\text { implementing in a } \\
\text { centralized system where } \\
\text { the area is divided into } \\
\text { subareas for a single RPA }\end{array}$ & $\begin{array}{l}\text { Possibility of finding the } \\
\text { optimal solution with the } \\
\text { changing number of RPA } \\
\text { and changing constants }\end{array}$ \\
\hline Disadvantages & $\begin{array}{l}\text { A monitoring mission } \\
\text { may fail in case of a } \\
\text { single RPA failure }\end{array}$ & $\begin{array}{l}\text { A monitoring mission may } \\
\text { fail in case of a single RPA } \\
\text { failure }\end{array}$ & $\begin{array}{l}\text { Long computation time } \\
\text { for } N>50\end{array}$ \\
\hline
\end{tabular}

The results of the conducted analyses show that for a multilevel control system for receiving the optimal decision that minimizes calculation time, both algorithms should be integrated. Taking into account the specificity of oil pollution surveillance mission, for the multi-RPA system there should be applied a centralized and a decentralized system. It means that in some cases the system can be operated without an operator after assigning tasks. In some cases, a feedback link with a dispatcher can be used.

The oil spill detection mission by using multi-RPA can be planned with a complex approach (Fig. 2) using the decision tree algorithm and ant colony algorithm.

A solution to the task of assigning for multi-RPA requires a lot of resources and time. For the practical implementation of oil detection mission, it is required to find a solution which is close to optimal in the defined probability interval. This restriction allows solving the problem of directing agents without complicated use of software resources. It is required to formulate initial input data for the algorithm taking into account that the RPA in the swarm fly with an average speed and the targets are connected with straight lines. This presentation of the mission is typically used for static types of objects. During the performance of oil pollution detection mission, some objects are moving, for example, ships.

To determine the meeting point of moving objects, the following functions are used: the function that determines the time of the movement of an object to one of the points on the straight line along which the object is moving; the function that determines the movement of the RPA to the point mentioned above [7]. This formulation of the mission task on the high level of control system allows to not use an imitation model for agents and objects. This approach allows simplifying the calculation of the length of straight lines and time of a flight for the RPA. For the complex approach algorithm on the high level, the matrix of time cost for all objects will be calculated. This matrix will be used for both centralized and ant colony algorithms. The distribution of objects between multi-RPA will be performed using both algorithms. The main restriction criteria for the distribution of RPA on the high level will be the number of targets $n$. The implementation of both algorithms in a multilevel system allows combining the effect on the high level. To reduce the dimensions of the matrix of objects after their analysis in the dynamic system, the solution can be found by using a heuristic algorithm for one RPA. The centralized system, when the monitoring tasks for each RPA are determined by the control center, allows to use RPA more efficiently in terms of flight endurance. 
Considering the high accuracy of the decision tree algorithm, it is possible to use it for the number of objects $n<20$ or if the area is split into subareas where the number of targets is $n<20$. The dynamic changes of an oil spill can be calculated according to the proposed model. The solution of oil spill monitoring and detection by multi-autonomous agents has a lot of solutions. On the high level, an oil pollution detection route for a group of RPA will be created. This information will be sent to the group which includes the RPA $R_{i}$. The optimal solution can be obtained with an algorithm where both the decision tree and ant colony algorithms are used. In this algorithm, two scenarios were implemented. In the first scenario the distribution of monitoring targets is computed by solving a graph of dynamic time cost matrix objects. In this scenario, the monitoring target set can be split to subsets. For each subset on the high level, the center of gravity of the targets will be calculated. In this subset, the targets will be connected by straight lines. This presentation allows using the closed graph $\mathrm{G}$ on the low level to find the optimal route for a single RPA. The task of the surveillance mission can be represented as the process of visiting all nodes on the graph $G(V, E)$ starting from the starting point and returning to the starting point [7]. In order to design swarm behaviour, a mathematical model must be provided for both individual agents and the whole swarm [25].

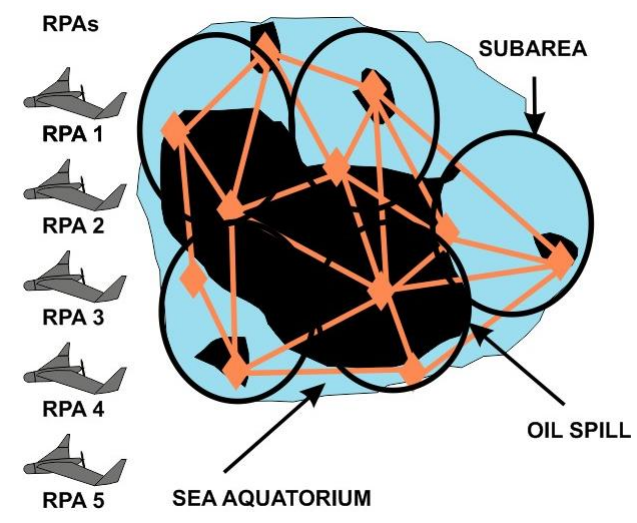

Fig. 2. A complex approach for oil detection.

In the second scenario, the ant colony algorithm is used for distributing dynamic time cost matrix between the RPA. In this algorithm, a restriction for pheromone evaporation can be implemented. In this approach, the amount of pheromone depends on the number of RPA using the straight line on the graph. Within a short period of time, the evaporation of pheromone can be taken into account. This option gives an opportunity to implement new recalculated matrix on the high level if the situation is changed in real time. Solutions obtained in both scenarios will be compared. The efficiency criterion $K_{1}$ and complexity criterion $K_{2}$ are used as criteria for assessing the efficiency of the considered algorithms (Fig. 3):

$$
K_{1}=\frac{T_{2}}{T_{1}},
$$

where

$T_{2} \quad$ Flight time during the monitoring of objects using the scenario of ant colony 
algorithm 2;

$T_{1} \quad$ Flight time during the monitoring of objects using scenario 1 of decision tree algorithm 1 .

$$
K_{2}=\frac{T_{\text {calc2 }}}{T_{\text {calc1 }}},
$$

where

$T_{\text {calc2 }} \quad$ Calculation time using the scenario of ant colony algorithm 2;

$T_{\text {calc1 }} \quad$ Calculation time using the scenario of decision tree algorithm 1.

In case if $K_{1}>1$ and $K_{2}>1$, the solution obtained as a result of implementing scenario 1 will be used as the optimal route for the group of RPA.

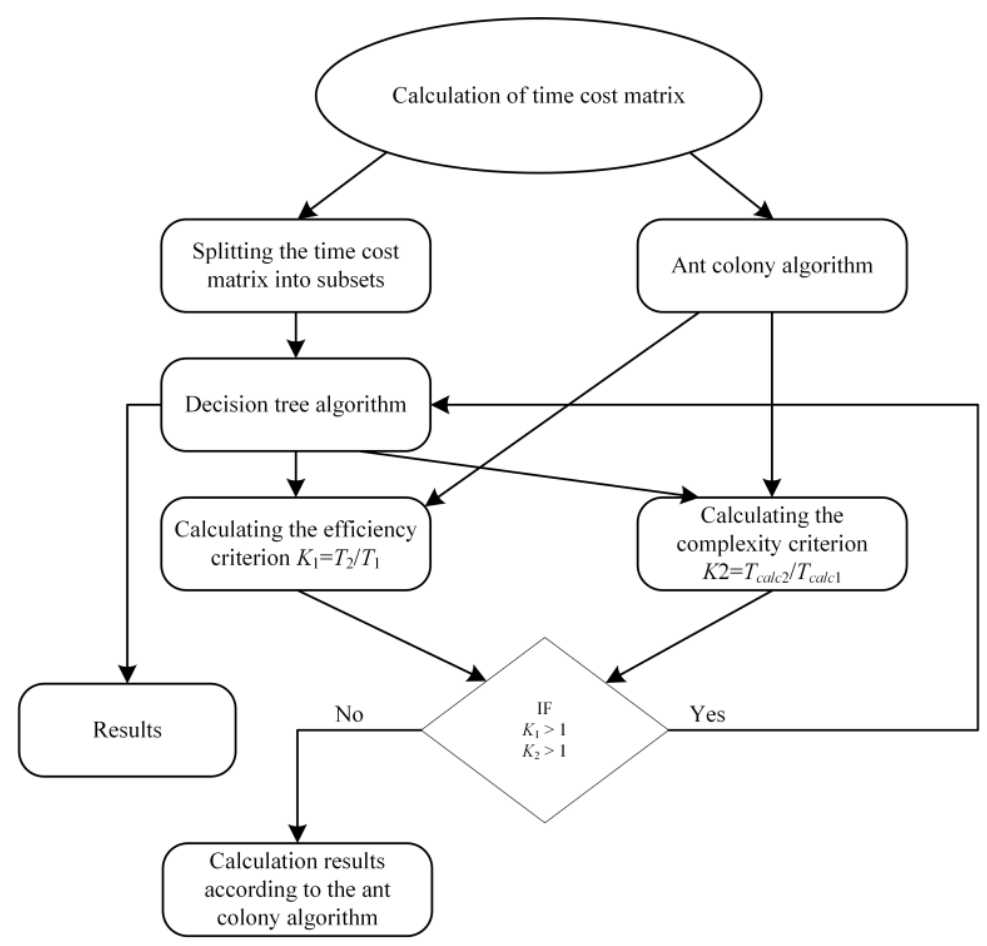

Fig. 3. The algorithm of time cost matrix calculation.

This approach allows to use the strengths of both algorithms and eliminate the weaknesses. The possibility to switch from one algorithm to another allows one to obtain a solution according to the dimension of the mission task and minimize the time required to solve the problem.

To minimize the flight time during the monitoring of objects, in the proposed algorithm the ant colony algorithm and decision tree algorithm are implemented in parallel. At the same time splitting the time cost matrix allows to apply a single-agent approach of the decision tree 
algorithm in parallel to the probabilistic ant colony algorithm, thus avoiding the drawbacks of each of the above algorithms and ensuring the achievement of high calculation accuracy. The developed algorithm can be used for calculating the optimal RPA flight path at various dimensions of the dynamic matrix of monitor objects.

The efficiency assessment of the developed algorithm in comparison with the decision tree and ant colony algorithms was carried out for various numbers of monitor objects and RPA used (Table 2).

TABLE 2. EFFICIENCY ASSESSMENT OF THE DEVELOPED ALGORITHM IN COMPARISON WITH THE DECISION TREE AND ANT COLONY ALGORITHMS

\begin{tabular}{|c|c|c|c|c|c|c|c|}
\hline \multicolumn{2}{|l|}{ Algorithm } & \multicolumn{2}{|c|}{ Decision tree algorithm } & \multicolumn{2}{|c|}{ Ant colony algorithm } & \multicolumn{2}{|c|}{ Developed algorithm } \\
\hline $\begin{array}{l}\text { Number of } \\
\text { objects } N\end{array}$ & $\begin{array}{l}\text { Number of } \\
\text { RPA } R\end{array}$ & $\begin{array}{l}\text { Flight time } \\
T, \mathrm{~s}\end{array}$ & $\begin{array}{l}\text { Calculation } \\
\text { time } T_{\text {calc }}, \mathrm{s}\end{array}$ & $\begin{array}{l}\text { Flight time } \\
T, \mathrm{~s}\end{array}$ & $\begin{array}{l}\text { Calculation } \\
\text { time } T_{\text {calc }}, \mathrm{s}\end{array}$ & $\begin{array}{l}\text { Flight time } \\
T, \mathrm{~s}\end{array}$ & $\begin{array}{l}\text { Calculation } \\
\text { time } T_{\text {calc }}, \mathrm{s}\end{array}$ \\
\hline 15 & 3 & 38.6 & 1 & 47.1 & 28 & 38.6 & 2 \\
\hline 20 & 4 & 33.1 & 2 & 33.5 & 29 & 33.1 & 2 \\
\hline 40 & 8 & 59.8 & 2 & 57.8 & 30 & 57.8 & 30 \\
\hline 90 & 18 & 78.0 & 2 & 60.0 & 31 & 60.0 & 31 \\
\hline
\end{tabular}

As it can be seen from Table 2, the developed algorithm is the most efficient, as it simultaneously uses the strengths of both the ant colony algorithm and decision tree algorithm when determining the minimum flight time during the monitoring of objects. The simulation results confirm the expediency of applying the developed algorithm for the purposes of optimizing flight routes during the monitoring of objects by a group of RPA.

\subsection{Sampling Method and Devices}

To increase the efficiency of monitoring with the help of a group of RPA, the latter have to be equipped with devices intended for taking oil pollution samples from the water surface; they also have to provide an opportunity to promptly analyse samples and transfer data in the online mode to both all the RPA participating in the mission and ground services. The previously known water sampling methods using RPA do not comply with the specified requirements [26].

Before starting the planned production activities, environmental impact assessment might be an obligatory aspect depending on the production type and volume [31]. During the development of an efficient and high-quality oil pollution monitoring solution, observations using the RPA were divided into the following main types:

- Permanent observations of the sea aquatorium areas with the aim of controlling the status and detection of pollution sources [27];

- Periodic surveys of large sea areas. For a rational monitoring over a given period, samples are taken throughout the area. In this case, permanent observations are made;

- A single sample take-up is taken in a specified area and is required for the confirmation of visual observation.

The proposed device, unlike the known ones, can be retracted into the body of the RPA, which ensures good aerodynamic characteristics during the mission. In addition to that, the measuring device ensures transfer of information about the degree of pollution in the online mode. The method and device for taking samples for the presence of oil pollution in the water aquatorium includes taking a sample from the water surface, preparation for 
transportation, determination of possible oil contamination using a measuring device, on-line data transmission, displaying the area of contamination on the electronic map and sample delivery to a specified location by using an RPA with fixed wings.

The device schematically shown in Fig. 4 comprises the RPA (1) with fixed wings equipped with a special sampler which was made as a metal sampler holder, mounted in the housing of the RPA, and a sampling device (2) mounted on the holder [28]. The design of the sampling device includes a cylindrical sampler with a solenoid valve with the spring (3) connected to the rod (4) with a sleeve with pistons (6) of the sampler chamber. The holes were made in the cylindrical body of the sampler for receiving water during the process of sampling. Inside the upper section, in the sampler upper piston, photoelectric cell (5) was mounted, while in the lower piston there was mounted lighting led (7).

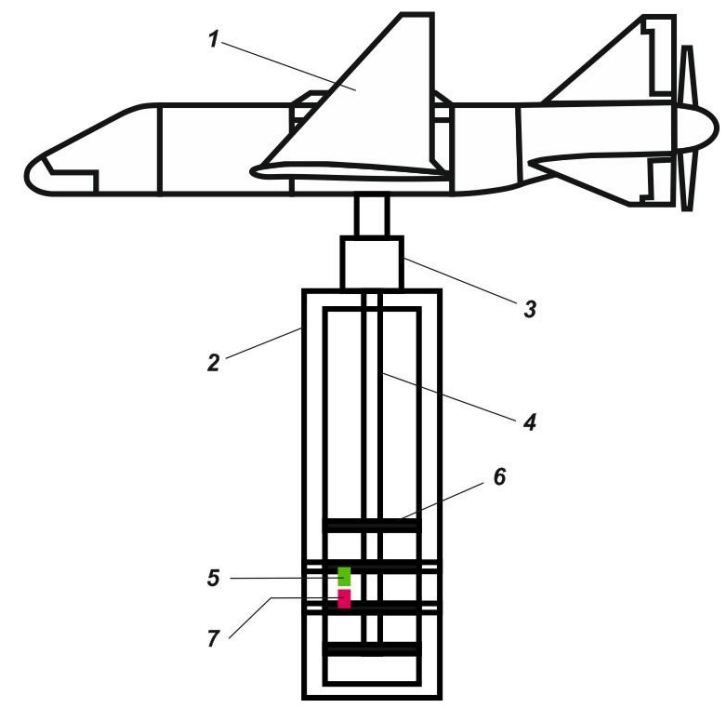

Fig. 4. Sampling device.

This solution provides an opportunity to carry out, after taking a sample, the control of a water sample for the presence of oil and contamination. The measuring cell consists of a photoelectric cell and a light diode which sends a signal to the electronic control module (ECM) of the RPA, when there is a presence of oil products (registered optical changes), and then a signal to the control center through the communication system. This solution allows using the coordinates where the RPA has taken samples, displaying the pollution area on a multi-level electronic map system.

The method is implemented as follows: the RPA is sent to a water sampling zone with known GPS coordinates. Upon the arrival of the RPA at a given zone, the electronic control module (ECM) gives a command to open the shutters of the sealed compartment of the sampling device. After opening the shutters, the ECM gives a command to turn the sample holder into a vertical position. The RPA reduces the flight height to touch the water surface with the sampling device. After touching the water surface with a container of the sampling device, the ECM gives a command to the solenoid valve to open a sleeve of the sampling device. Water gets inside the sampling device. The ECM gives a command to the solenoid valve to close the sleeve of the sampling device. 
The sleeve pistons seal the taken sample inside the sampling device. The ECM gives a command to turn the sample holder into a horizontal position. Upon the completion of sample holder turning, the ECM gives a command to close the shutters of the sealed compartment of the sampling device. The sample is taken and transported automatically. The measuring cell consists of a photoelectric cell and a light diode; when there is a presence of oil products (registered optical changes), it sends a signal to the electronic control module ECM of the RPA and then it sends a signal to the control center through the communications system; simultaneously, the coordinates of the sampling point are sent. The received information about the pollution is displayed on a multi-level electronic map.

\section{Conclusions}

This paper describes the process of solving the problem of assigning tasks to multi Remote Piloted Aircraft (RPA) performing an oil pollution detection mission. The limitations of centralized and decentralized systems can be minimized using a complex approach. The algorithm is implemented without any communication among the RPAs. Taking into account the specificity of oil pollution detection mission, a control cycle of the system was developed on the high level. It allows to create a surveillance task for discrete events, such as an oil spill. The objective is to carry out an analysis of remote oil spill detection by using multi-Remote Piloted Aircraft. An oil spill spread model, which is used for creating a matrix of objects for the RPA, was developed. The multi-RPA system provides a reliable detection of an oil spill with significant advantages over other existing single-RPA methods. A complex algorithm for oil spill monitoring and detection was developed. The decision tree algorithm and ant colony algorithm were analysed through an analysis. Using the results of the analysis, an algorithm with two scenarios was developed: the decision tree and ant colony. This approach allows to eliminate the disadvantages and enhance the strengths of both algorithms. The complex solution accuracy will be improved by determining the decision tree algorithm. The sampling device significantly improves the quality of monitoring with the help of multi-RPA.

\section{REFERENCES}

[1] Urbahs A., Zavtkevics V. Oil Pollution Monitoring of Sea Aquatorium Features with Using Unmanned Aerial Vehicles. Presented at the $18^{\text {th }}$ International Conference Kaunas, Lithuania 2014:75-78.

[2] Brekke C., Solbergb A. Oil spill detection by satellite remote sensing [Online]. [Accessed 30.11.2017]. Available: https://pdfs.semanticscholar.org/af8d/474288595d6bb7b1b90e530c16ca17d647c8.pdf

[3] Muttin F. Modeling of captive Unmanned Aerial System tele detecting oil pollution on sea surface. John Wiley \& Sons, 2014. https://doi.org/10.1002/9781119003021.ch7

[4] Urbahs A., Jonaite I. Features of the use of unmanned aerial vehicles for agriculture applications. Aviation 2013:17:170-175. https://doi.org/10.3846/16487788.2013.861224

[5] Fingas M., Brown C. Review of Oil Spill Remote Sensing. Marine Pollution Bulletin 2014:83:9-23. https://doi.org/10.1016/j.marpolbul.2014.03.059

[6] Urbahs A., Zavtkevics V. Remote Piloted Aircraft using for sampling of oil spill. Presented in the proceedings of Transport Means 2017. The $21^{\text {st }}$ international scientific conference. Part 2, Kaunas, Lithuania, 2017.

[7] Urbahs A., Zavtkevics V. Remotely Piloted Aircraft route optimization when performing oil pollution monitoring of the sea aquatorium. Aviation 2017:21:170-175. https://doi.org/10.3846/16487788.2017.1344139

[8] Dijkstra E. A note on two problems in connection with graphs. Numerische Mathematik 1959:1:269-271. https://doi.org/10.1007/BF01386390

[9] Dantzig G., Fulkerson R., Johnson S. Solution of a Large-Scale Traveling-Salesman Problem. Journal of the Operations Research Society of America 1954:2:393-410. https://doi.org/10.1287/opre.2.4.393

[10] Adubi S., Misra S. A comparative study on the ant colony optimization algorithms. Presented in the $11^{\text {th }}$ International Conference on Electronics, Computer and Computation (ICECCO), Abuja, Nigeria, 2014. 
[11] Walter B., Sannier A., Reiners D., Oliver J. UAV Swarm Control: Calculating Digital Pheromone Fields with the GPU. The Journal of Defense Modeling and Simulation Applications, Methodology, Technology 2006:3:167-176. https://doi.org/10.1177\%2F154851290600300304

[12] Niccolini M., Pollini L., Innocenti L. Cooperative Control for Multiple Autonomous Vehicles Using Descriptor Functions. The Journal of Sensor and Actuator Networks 2014:3:26-43. https://doi.org/10.3390/jsan3010026

[13] Eaton C., Chong K., Maciejewski A. Multiple-Scenario Unmanned Aerial System Control: A Systems Engineering Approach and Review of Existing Control Methods. Aerospace 2016:3. http://dx.doi.org/10.3390/aerospace3010001

[14] Rubio J. C., Vagners J., Rysdyk R. Adaptive path planning for autonomous UAV oceanic search missions. Presented at the Intelligent Systems Technical Conference, Chicago, USA, 2004. https://doi.org/10.2514/6.2004-6228

[15] Cottam R., Ranson W., Vounckx R. Autocreative hierarchy II: dynamics self-organization, emergence and level-changing. Presented at the International Conference on Integration of Knowledge Intensive Multi-Agent Systems, Cambridge, USA, 2003. https://doi.org/10.1109/KIMAS.2003.1245134

[16] Sun A., Liu H. Cooperative UAV Search for Moving Targets Using a Modified Diffusion Uncertainty Model, 2009. http://dx.doi.org/10.2514/6.2009-5779

[17] Cummings M., Bruni S., Mercier S., Mitchell P. Automation Architecture for Single Operator, Multiple UAV Command and Control. International C2 Journal 2007.

[18] AeroVations Associates. Autonomous Civil Unmanned Aircraft Systems Software Quality Assessment and Safety Assurance. Autonomous Unmanned Aircraft Systems 2914, 2007.

[19] Belta C., Kumar V. Abstractions and control for groups of robots. IEEE Transactions on Robotics 2004:20:865-875. https://doi.org/10.1109/TRO.2004.829498

[20] Bertuccelli L., How J. Robust UAV Search for Environments with Imprecise Probability Maps. Presented at the IEEE Conference on Decision and Control, and the European Control Conference, Seville, Spain, 2005. https://doi.org/10.1109/CDC.2005.1583068

[21] Zhang C., Pei H. Oil Spills Boundary Tracking Using Universal Kriging and Model Predictive Control by UAV. Presented at the $11^{\text {th }}$ World Congress on Intelligent Control and Automation, Shenyang, China, 2014.

[22] Hirsch M. J., Schroeder D. On the Decentralized Cooperative Control of Multiple Autonomous Vehicles. Handbook of Unmanned Aerial Vehicles. Springer, 2015. https://doi.org/10.1007/978-90-481-9707-1_112

[23] Beni G. From swarm intelligence to swarm robotics. Swarm Robotics. Berlin: Springer, 2004. https://doi.org/10.1007/978-3-540-30552-1_1

[24] Fallahi K., Leung H., Chandana S. An Integrated ACO-AHP Approach for Resource Management Optimization. Presented at the IEEE International Conference on Systems, Man, and Cybernetics, San Antonio, USA, 2009.

[25] Koparan C., Koc A., Privette C., Sawyer C. Evaluation of a UAV-Assisted Autonomous water Sampling. Water 2018:10(5):655. https://doi.org/10.3390/w10050655

[26] Soysal O., Sahin E. A macroscopic model for self-organized aggregation in swarm robotic systems. Swarm Robotics. Berlin: Springer, 2007:27-42. https://doi.org/10.1007/978-3-540-71541-2_3

[27] Urbahs A., Zavtkevics V. Unmanned aerial vehicle for collecting samples from the surface of water. EU patent EP3112840 (A1).

[28] Urbahs A., Zavtkevics V. Water sampling method of oil pollution and for analysis using unmanned aerial vehicle with fixed wings and device for method perform. LV patent application P-15-88 2015-08-20.

[29] Kittipongvises S. Assessment of Environmental Impacts of Limestone Quarrying Operations in Thailand. Environmental and Climate Technologies 2017:20:67-83. https://doi.org/10.1515/rtuect-2017-0011

[30] Avotniece Z., Briede A., Klavins M., Aniskevich S. Remote Sensing Observations of Thunderstorm Features in Latvia. Environmental and Climate Technologies 2017:21:28-46. https://doi.org/10.1515/rtuect-2017-0014

[31] Dagiliute R., Juozapaitiene G. Stakeholders in the EIA Process: What is Important for Them? The Case of Road Construction. Environmental and Climate Technologies 2018:22:69-82. https://doi.org/10.2478/rtuect-2018-0005

[32] Bajcinovci B. Environment Quality: Impact from Traffic, Power Plant and Land Morphology, a Case Study of Prishtina. Environmental and Climate Technologies 2017:19:65-74. https://doi.org/10.1515/rtuect-2017-0006

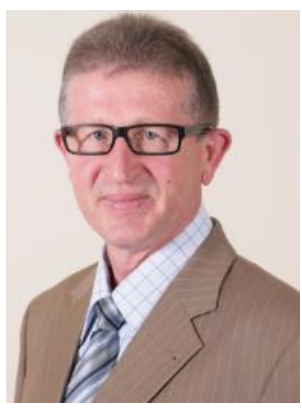

Aleksandrs Urbahs', Dr. habil. sc. ing. Graduated from the Faculty of Mechanical Engineering, Riga Civil Aviation Engineering Institute in 1981. In 1986 he was awarded a Dr. sc. ing. degree by the same faculty. In 1997 he was awarded a Dr. habil. sc. ing. degree by Riga Aviation University. In the period from 1989 to 1999 - Vice Dean and Dean of the Faculty of Mechanical Engineering, Riga Aviation University. Since 1999 - full-time Professor at Riga Technical University. In the period from 1999 to 2012 - Head of the Institute of Transport Vehicle Technologies at Riga Technical University. Since 2012 - Head of the Institute of Aeronautics at Riga Technical University. Since 2017 he has been Associate Member of Latvian Academy of Science. He holds more than 34 patents and has published more than 285 scientific papers. His fields of research: aeronautics, remotely piloted aircraft systems, transport systems and logistics, fatigue cracks, non-destructive testing, nanomaterials, structural materials.

ORCID iD: https://orcid.org/0000-0003-1307-1791 


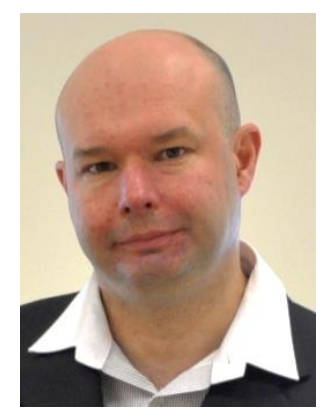

Vladislavs Zavtkevics has been a Doctoral Student at the Institute of Aeronautics, Riga Technical University since 2013. In 2010 he obtained a degree of Master of Science at the Latvian Maritime Academy. He graduated from the Latvian Maritime Academy in 2004 and obtained specialisation Engineer - Ship's Navigator.

Work experience: 2000-2005 Navigator of Merchant vessels. From 2005 till present time Captain on Latvian Coast Guard patrol ship. From August 2009 he is working as Lecturer in LMA on course "Cargo transportation technologies".

His field of research: Unmanned Underwater Vehicles, Ships cargo handling and stowage procedures; Marine Environment protection, Ship' Theory.

ORCID iD: https://orcid.org/0000-0002-3210-9372 